\title{
Transferrin mutations at the glycosylation site complicate diagnosis of congenital disorders of glycosylation type I
}

\author{
Mailys Guillard · Yoshinao Wada • Hana Hansikova • Isao Yuasa • Katerina Vesela • \\ Nina Ondruskova $\cdot$ Machiko Kadoya $\cdot$ Alice Janssen • \\ Lambertus P. W. J. Van den Heuvel • Eva Morava • Jiri Zeman • Ron A. Wevers • \\ Dirk J. Lefeber
}

Received: 15 December 2010 / Revised: 17 February 2011 / Accepted: 21 February 2011 /Published online: 23 March 2011

(C) The Author(s) 2011. This article is published with open access at Springerlink.com

\begin{abstract}
Congenital disorders of glycosylation (CDG) form a group of metabolic disorders caused by deficient glycosylation of proteins and/or lipids. Isoelectric focusing (IEF) of serum transferrin is the most common screening method to detect abnormalities of protein $\mathrm{N}$-glycosylation. On the basis of the IEF profile, patients can be grouped into CDG type I or CDG type II. Several protein variants of transferrin are known that result in a shift in isoelectric point $(\mathrm{p} I)$. In some cases, these protein variants co-migrate with transferrin glycoforms, which complicates interpretation. In two patients with abnormal serum transferrin IEF profiles, neuraminidase digestion and subsequent IEF showed profiles suggestive of the diagnosis of CDG type I. Mass spectrometry of tryptic peptides of immunopurified transferrin, however,
\end{abstract}

Communicated by: Verena Peters

Competing interest: None declared.

M. Guillard - A. Janssen • L. P. W. J. Van den Heuvel •

R. A. Wevers $\cdot$ D. J. Lefeber $(\bowtie)$

Institute for Genetic and Metabolic Disease,

Department of Laboratory Medicine / Department of Neurolog,

Radboud University Nijmegen Medical Centre,

Geert Grooteplein 10,

6525 GA Nijmegen, The Netherlands

e-mail: d.lefeber@neuro.umcn.nl

M. Guillard · D. J. Lefeber

Department of Neurology,

Radboud University Nijmegen Medical Centre,

Nijmegen, The Netherlands

E. Morava

Department of Paediatrics,

Radboud University Nijmegen Medical Centre,

Nijmegen, The Netherlands revealed a novel mutation at the $\mathrm{N}$-glycan attachment site. In case 1 , a peptide with mutation p.Asn630Thr in the 2nd glycosylation site was identified, resulting in an additional band at disialotransferrin position on IEF. After neuraminidase digestion, a single band was found at the asialotransferrin position, indistinguishable from CDG type I patients. In case 2, a peptide with mutation p.Asn432His was found. These results show the use of mass spectrometry of transferrin peptides in the diagnostic track of CDG type I.

\section{Abbreviations \\ CDG Congenital disorders of glycosylation \\ IEF Isoelectric focusing \\ LC-MS Liquid chromatography mass spectrometry}

\section{Y. Wada $\cdot$ M. Kadoya}

Osaka Medical Center and Research Institute

for Maternal and Child Health,

Osaka, Japan

I. Yuasa

Division of Legal Medicine, Tottori University,

Yonago, Japan

H. Hansikova $\cdot$ K. Vesela $\cdot$ N. Ondruskova $\cdot J$. Zeman

Department of Pediatrics and Adolescent Medicine,

First Faculty of Medicine, Charles University in Prague and General University Hospital in Prague,

Prague, Czech Republic 
MALDI Matrix assisted laser desorption/ionisation TOF Time-of-flight

\section{Introduction}

Congenital disorders of glycosylation (CDG) comprise a group of rare inherited metabolic disorders caused by deficient glycosylation of proteins and/or lipids. For Nglycan biosynthesis, a lipid-linked oligosaccharide is synthesized in the endoplasmatic reticulum, and transferred en bloc to a nascent protein. N-glycans are exclusively transferred to an asparagine in the consensus sequence Asn$\mathrm{Xxx}$-Ser/Thr, where $\mathrm{Xxx}$ is any amino acid except proline. The glycosylated protein is then transferred to the Golgi for further processing of the glycans. Defects in the biosynthesis and transfer of the lipid-linked oligosaccharide result in the loss of whole $\mathrm{N}$-glycans on glycoproteins, resulting in a CDG type I (CDG-I).

Diagnostic screening of CDG is performed by isoelectric focusing (IEF) of transferrin. In its most abundant form, this serum glycoprotein carries two complex N-glycans attached to Asn432 and Asn630. Each N-glycan is terminated by two negatively charged sialic acids. Defects in the biosynthesis of $\mathrm{N}$-glycans in the endoplasmatic reticulum cause a loss of one or two complete N-glycans, resulting in a typical IEF pattern of CDG-I, characterized by increased asialo- and disialotransferrin bands, and decreased tetrasialotransferrin. Secondary causes of hypoglycosylation such as galactosemia or fructosemia may cause an identical pattern and therefore must be excluded. Several transferrin protein polymorphisms result in a shifted IEF pattern, caused by $\mathrm{p} I$ differences of the polypeptide chain (Fujita et al. 1985; Kamboh and Ferrell 1987). Variant TF $C$ is the most common form of transferrin, the anodic variant $\mathrm{B}$ and cathodic variant $\mathrm{D}$ are less common. In particular, heterozygous combinations of $\mathrm{C}$ and $\mathrm{D}$ variants may lead to misinterpretation in CDG diagnosis (Fujita et al. 1985; Marklova and Albahri 2009). Nevertheless, these polymorphisms can be identified by neuraminidase treatment of serum, resulting in an IEF pattern with two distinct asialotransferrin bands (Fig. 1, lane 5).

To improve diagnostics of $\mathrm{CDG}$, mass spectrometric methods for glycoprotein analysis are being developed because of their higher sensitivity and specificity (Hahn et al. 2006; Lacey et al. 2001; Wada 2006; Wada 2007). Liquid chromatography-mass spectrometry (LC-MS) of immunopurified transferrin was proven to be a sensitive screening method for CDG-I, showing distinct peaks for fully glycosylated transferrin, and transferrin lacking one or two oligosaccharide chains (Bunkenborg et al. 2004; Hulsmeier et al. 2007; Lacey et al. 2001; Wada et al. 1992). For CDG type II with abnormal processing of glycans, analysis of enzymatically removed glycans can be diagnostic (Guillard et al. 2011).

Here, we present the application of MS of transferrin peptides in the diagnosis of CDG and reveal two novel transferrin mutations in the N-glycan attachment site.

\section{Patients and methods}

\section{IEF and SDS-PAGE of transferrin}

IEF of transferrin was performed as described in two cases. Case 1 was suspected of CDG due to a clinical presentation of cyclic vomiting accompanied with ketosis and metabolic acidosis and the appearance of atypical fat pads and inverted nipples at the age of seven. Hemoglobin and mean corpuscular volume were normal. Case 2 was a healthy donor for blood transfusion, who participated in a largescale screening for transferrin variants in populations of Japan and New Zealand (Yuasa et al. 1987). His mother was also healthy.

Five $\mu \mathrm{L}$ of plasma was incubated for 30 min with equal volumes of $6.7 \mathrm{mM} \mathrm{Fe}(\mathrm{III})$ citrate and $0.17 \mathrm{mM}$ $\mathrm{NaHCO}_{3}$ (Fluka, Buchs, Switzerland) in ultrapure water, then diluted ten times in saline. Two $\mu \mathrm{L}$ of each sample was transferred to a hydrated immobilin dry gel (GE Healthcare, Buckinghamshire, UK) with Servalyt, $\mathrm{pH}$ 5-7
Fig. 1 Transferrin isoelectric focusing profiles before and after neuraminidase incubation. Lane 1 and 1': control, lane 2 and 2': PMM2-CDG, lane 3 and 3': case 1 , lane 4 and 4 ': mother of case 1 , lane 5 and 5': case 2, lane 6 and 6 ': mother of case 2 , lane 7 and 7': patient with a known "D variant" polymorphism. Left and right of the figure are the numbers of sialic acids present on transferrin $\mathrm{C}$ variant

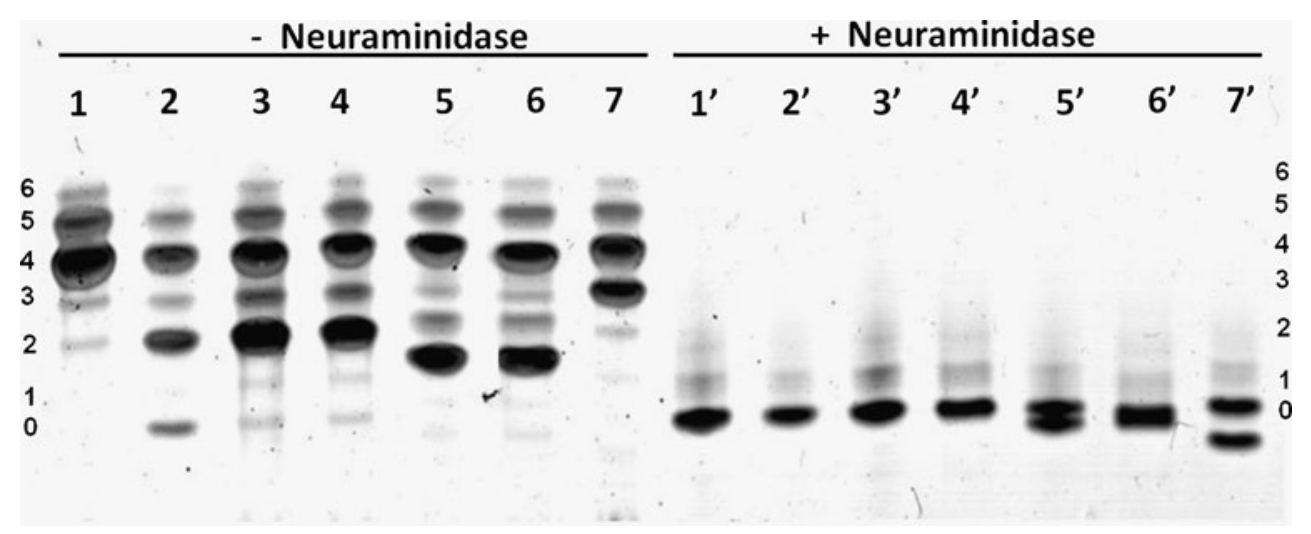


(Serva, Heidelberg, Germany) and run on a PhastSystem (GE Healthcare). Different isoforms of transferrin were detected by immunoprecipitation in the gel with $60 \mu \mathrm{L}$ rabbit-anti-human transferrin antibody per gel ( $8.5 \mathrm{~g} / 1$; Dako, Glostrup, Denmark) for $30 \mathrm{~min}$, followed by overnight washing in saline, fixation with $20 \%(\mathrm{w} / \mathrm{v})$ trichloracetic acid and Coomassie blue staining.

Removal of sialic acids was achieved by incubating $3 \mu \mathrm{L}$ of plasma for $30 \mathrm{~min}$ with the same volume of $6.7 \mathrm{mM} \mathrm{Fe}$ (III) citrate and $0.17 \mathrm{mM} \mathrm{NaHCO}$ (Fluka) in water, prior to addition of $15 \mathrm{mU}$ of neuraminidase (Roche diagnostics, Mannheim, Germany) and incubation for two hours at $37^{\circ} \mathrm{C}$. IEF was then performed identically to non-treated samples.

Prior to SDS-PAGE analysis, plasma samples were centrifuged and diluted 100-fold with ultrapure water. Three $\mu \mathrm{L}$ diluted sample was mixed with $100 \mu \mathrm{L}$ sample buffer (10 mM Tris-HCl, pH 8.0, 1 mM EDTA, 2.5\% SDS, $2 \%$ DTT, and $0.01 \%$ bromophenol blue) and heated for $5 \mathrm{~min}$ at $95^{\circ} \mathrm{C}$. The samples were then applied to a $12.5 \%$ Phastgel (GE Healthcare) and the proteins separated on a Phastsystem (GE Healthcare). After separation, the proteins were detected by western blotting using a primary rabbit anti-human transferrin antibody (Dako) and a secondary goat anti-rabbit peroxidase conjugated antibody, followed by electrochemiluminescence detection.

\section{Matrix assisted laser desorption/ionization-MS} (MALDI-MS) of transferrin

The purified transferrin was desalted by a ZipTip C4 (Millipore, Bedford, MA) and analysed by MALDI mass spectrometry using a Voyager DE Pro MALDI-time-of-flight mass spectrometer with a nitrogen pulsed laser $(337 \mathrm{~nm})$ (Applied Biosystems, Foster City, CA) (Wada et al. 1994). The sample matrix was $10 \mathrm{mg} / \mathrm{mL}$ sinapinic acid in a $0.1 \%$ $(\mathrm{v} / \mathrm{v})$ TFA and $30 \%(\mathrm{v} / \mathrm{v})$ acetonitrile solution. The measurements were carried out in positive ion and linear TOF mode.

For electrospray MS of peptides, transferrin was dissolved in a solution of $6 \mathrm{M}$ guanidine, $0.25 \mathrm{M}$ Tris- $\mathrm{HCl}, \mathrm{pH}$ 8.0 , reduced with $0.13 \mathrm{M}$ dithiothreitol at $50^{\circ} \mathrm{C}$ for $1 \mathrm{~h}$ and then S-carbamidomethylated with $0.22 \mathrm{M}$ iodoacetamide for $30 \mathrm{~min}$ at room temperature. Tryptic peptides were desalted by ZipTip C18 (Millipore), dissolved in a $0.1 \%$ formic acid and $50 \%(\mathrm{v} / \mathrm{v})$ acetonitrile solution and were directly infused into an LTQ XL ion trap mass spectrometer (Thermo-Fisher Scientific, San Jose, CA) using a nanospray tip. The CID MS/MS was carried out with helium gas and the spectra were acquired by 100 scans.

Genetic analysis

DNA from case 1 and his mother was isolated from whole blood samples by the commonly used salt-out method for
DNA isolation. DNA of other family members was isolated from archived dried blood spots on filter paper using the QIAamp DNA Micro Kit (Qiagen, Valencia, CA). The isolation process was performed according to the recommended manufacturer protocols. The primers were designed to the intronic regions surrounding the 17 protein coding exons of the transferrin gene ( $T F$ gene: OMIM 190000; NCBI gene ID: 7018; NG_013080.1; ENSG00000091513). Primer sequences are available upon request. The fragments were amplified using the Combi PPP Master Mix (Top-Bio s.r.o; Praha, Czech Republic) and purified by gel extraction using Wizard SV Gel and PCR clean-Up System (Promega, Madison, WI). All fragments were sequenced on ABI PRISM 3100-Avant Genetic Analyzer (Applied Biosystems, Carlsbad, CA) using the Big Dye Terminator v.3.1 Cycle Sequencing Kit (Applied Biosystems) according the recommended manufacturer protocols. Sequence analyses were performed using SeqScape Software v2.5 (Applied Biosystems). The nucleotide numbering follows cDNA numbering with +1 corresponding to the A of the ATG translation codon in the reference sequence. The initiation codon is codon 1. For restriction analyses of the c.1889A $>\mathrm{C}$ mutation, the HpyCH4IV (New England BioLabs, Ipswich, MA) restriction endonuclease was used according to the manufacturer protocols of the enzyme.

\section{Results}

Clinical suspicion of CDG in case 1 triggered us to perform IEF of transferrin, which showed a type I profile (Fig. 1, lane 3) with increased disialotransferrin and decreased tetrasialotransferrin. Neuraminidase treatment resulted in a single band corresponding to asialotransferrin, suggesting that the double band in the untreated sample was not the result of a protein variant (Fig. 1, lane 3'). IEF of serum transferrin from case 2 and his mother showed two major bands in a 1:1 ratio with one band at the level of tetrasialotransferrin and one band just below disialotransferrin (Fig. 1, lanes 5 and 6). After neuraminidase treatment, two bands were visible at the asialotransferrin position (Fig. 1, lanes 5' and 6'). The distance between these two bands is much smaller than between the bands of sialylated transferrin.

Additional information was obtained by SDS-PAGE analysis of transferrin. In controls, transferrin SDS-PAGE shows a single band around $79 \mathrm{kDa}$ corresponding to the glycoprotein with two N-glycans (Fig. 2). The transferrin of a PMM2-CDG (CDG-Ia) patient showed bands with nonglycosylated transferrin at $75 \mathrm{kDa}$, transferrin with one Nglycan at $77 \mathrm{kDa}$ and the fully glycosylated transferrin at $79 \mathrm{kDa}$ (Wada et al. 1994). In cases 1 and 2, two bands of equal intensity were observed at $77 \mathrm{kDa}$ and $79 \mathrm{kDa}$. This 
Fig. 2 MALDI-TOF profile of immunopurified transferrin, carrying $1(\mathrm{~m} / z 77.3 \mathrm{kDa})$ or 2 $(\mathrm{m} / z 79.6 \mathrm{kDa}) \mathrm{N}$-glycan chains. Dotted line: control, continuous line: case 1 . The gray boxes represent the glycosylation consensus sequences of the transferrin protein. Right: SDS-PAGE of serum transferrin: $\mathrm{C}=$ control, $\mathrm{C} 1=$ case 1 , C1.M. $=$ mother of case 1

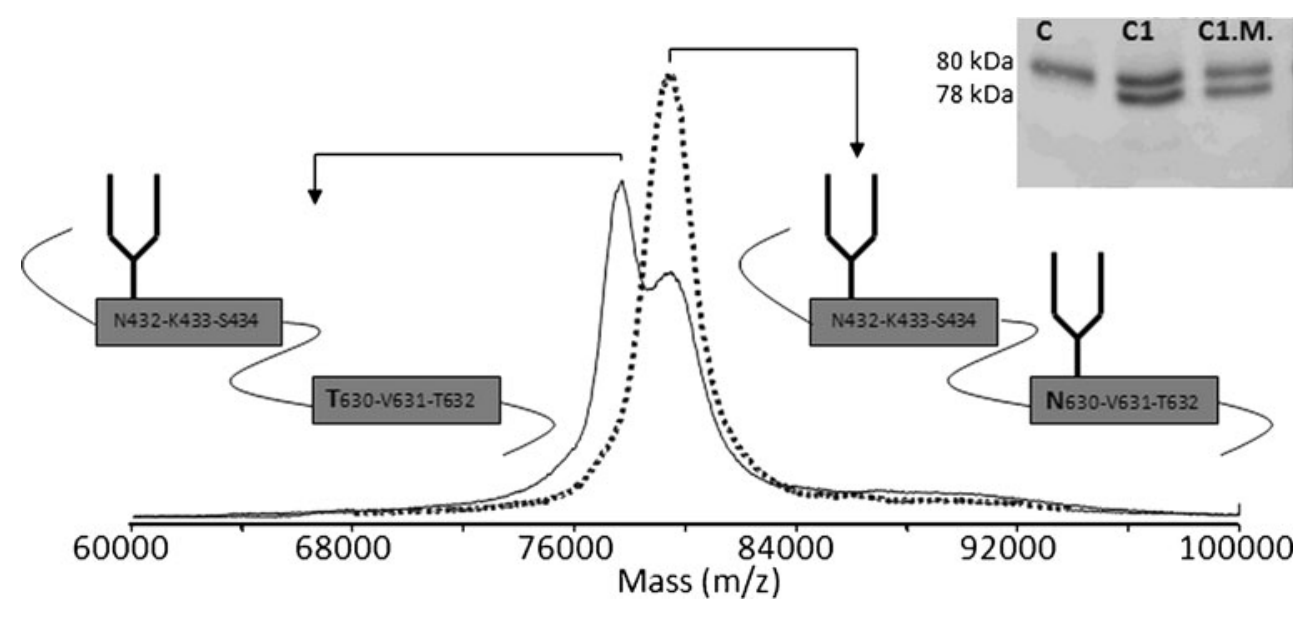

finding was confirmed by MALDI-MS of whole transferrin (Wada et al. 1994). Instead of a single peak at $79.6 \mathrm{kDa}$, peaks were observed at $77.3 \mathrm{kDa}$ and $79.6 \mathrm{kDa}$, corresponding to transferrin with one and two N-glycans, respectively (Fig. 2).

Despite these indications for true CDG-I profiles, a number of clues suggested otherwise. On transferrin IEF, the disialioand tetrasialotransferrin bands were present in a 1:1 ratio, while asialotransferrin was normal $(<3 \%)$. In CDG-I patients, such severe profiles are commonly associated with an increase of asialotransferrin. IEF of thyroxin binding globulin (TBG), another serum glycoprotein used for screening of $\mathrm{CDG}$, showed a normal pattern (data not shown). Additionally, transferrin IEF and SDS-PAGE in serum of the healthy mothers revealed similar patterns to their children's (Fig. 1, lanes 4 and 6; Fig. 2). In contrast to the results after neuraminidase digestion, these findings were indicative of a protein variant.

In order to obtain more insight into the structure of the transferrin protein in cases 1 and 2, transferrin was immunopurified from serum, trypsinized, and the peptides were analyzed by mass spectrometry. Glycopeptides, known to be present in the normal transferrin, were detected. In addition, a thus-far unreported nonglycosylated peptide was found in case 1 as doubly charged ion at $m / z 1252.1$ and triply charged ion at $\mathrm{m} / \mathrm{z}$ 835.0. MS/MS analysis revealed the peptide

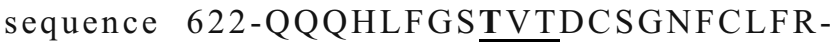
642 , corresponding to the non-glycosylated glycopeptide 2 with a heterozygous p.Asn630Thr change (Fig. 3). This asparagine is the second N-glycosylation site of transferrin (underlined). In case 2, an unknown doubly charged ion with $\mathrm{m} / \mathrm{z} 750.4$ was found. MS/MS sequencing indicated a $\mathrm{p}$. Asn432His change in the first glycosylation site: 421CGLVPVLAENYHK-433 (Fig. 4).

Thus, in both cases, MS/MS analysis of transferrin peptides showed a heterozygous amino acid change in one of the N-glycan attachment sites, resulting in a transferrin molecule with a single N-glycan. As a result, the mutated transferrin will lack two sialic acids and therefore be located at the disialotransferrin level on IEF. The imidazole group of the histidine in case 2 causes a slight $\mathrm{p} I$ difference between the common disialotransferrin and the mutated form.

For case 1, sequencing of the $T F$ gene revealed the heterozygous c.1889A $>\mathrm{C}$ mutation, thereby confirming the p.Asn630Thr alteration at the amino acid level. RFLP analysis in family members of case 1 showed the heterozygous mutation in the mother of case 1 , her sister and the grandmother. This p.Asn630Thr mutation was not found in 130 controls.

Case 2 was not available for further investigations at the molecular level. Yet, based on the amino acid sequence, a c.1294A > C mutation may have occurred, resulting in the substitution from Asn (AAT) to His (CAT).

\section{Discussion}

In the diagnosis of $\mathrm{CDG}$, it is important to exclude secondary causes of hypoglycosylation of transferrin, as well as transferrin variants which show an IEF pattern suggestive of CDG. In this study, we showed that the current method to identify a protein variants by treatment of serum samples with neuraminidase can be misleading when the mutation is located in one of the amino acids of the consensus sequence of N-glycosylation for transferrin. The loss of the N-glycan in half of the transferrin proteins in serum resulted in a pattern with equal amounts of disialoand tetrasialotransferrin. Although this resembles a CDG type I pattern, the approximate 1:1 ratio between the two isoforms in combination with low levels of asialotransferrin $(<3 \%)$, should trigger one to consider a mutation in one of the glycosylation sites. If neuraminidase treatment is inconclusive, IEF of another glycoprotein, like TBG, may provide additional information about a generalized glycosylation defect. When serum of the patient's parents is 


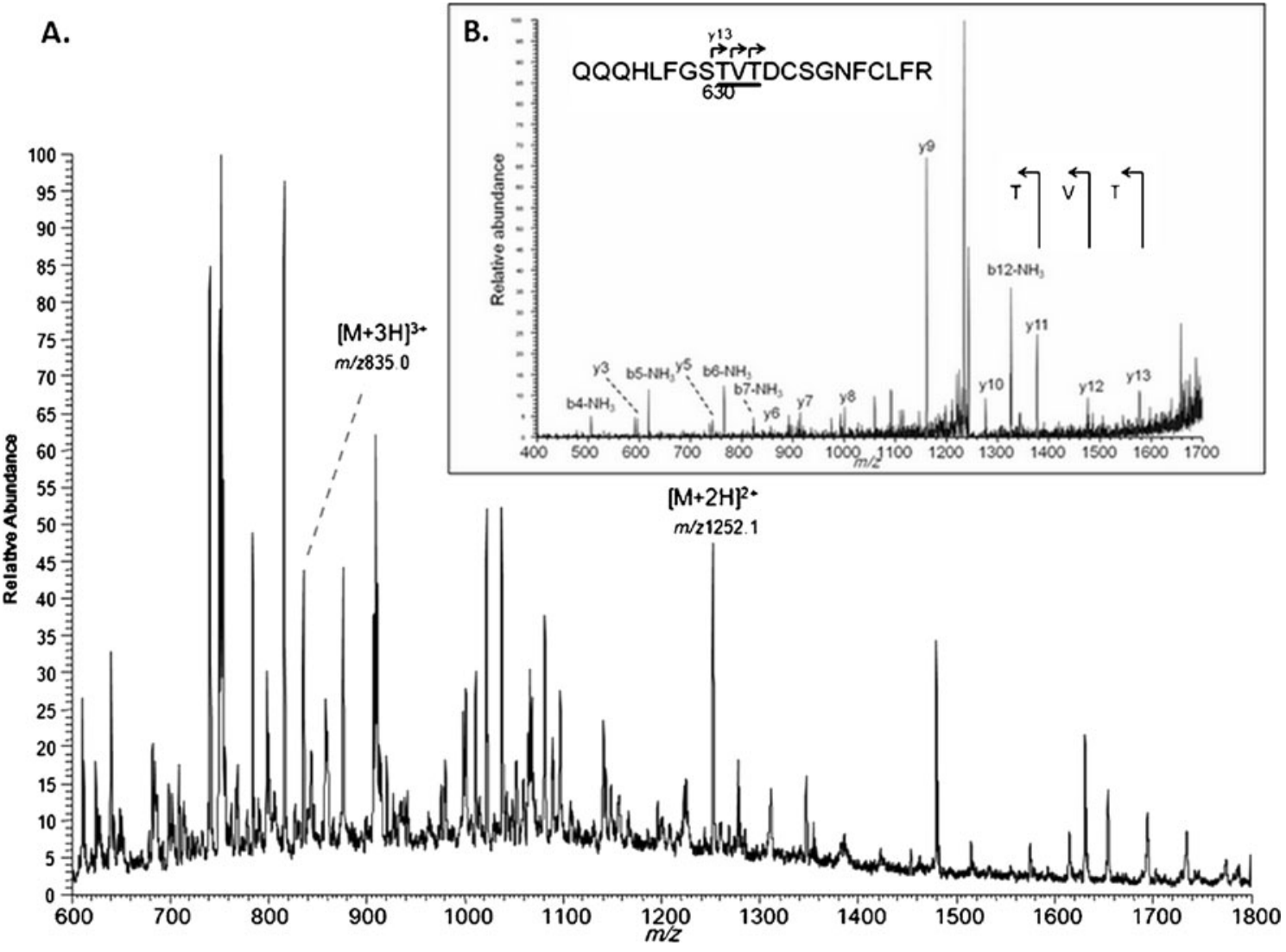

Fig. 3 Analysis of transferrin peptides in case 1. a identification of doubly- and triply charged species, not present in controls. b MS/MS analysis of $\mathrm{m} / \mathrm{z} 1252.1$ indicates a p.N630T mutation in the second transferrin glycosylation site, thus peptide 622QQQHLFGSNVTDCSGNFCLFR-642 is mutated to 622QQQHLFGSTVTDCSGNFCLFR-642

Wada 2007; Guillard et al. 2011). Here, we show that the application of MS of transferrin (glyco)peptides reveals a non-glycosylated peptide with a mutated N-glycosylation consensus sequence Asn-Xxx-Thr/Ser. Alternatively, identification of non-glycosylated peptides with normal sequence
Fig. 4 Identification of a protein mutation in case 2 . MS/MS analysis of $\mathrm{m} / \mathrm{z} 750.4$ indicated a p.N432H mutation in the first transferrin glycosylation site in case 2 , thus peptide 421-CGLVPVLAENYNK-433 is mutated to 421 CGLVPVLAENYHK-433

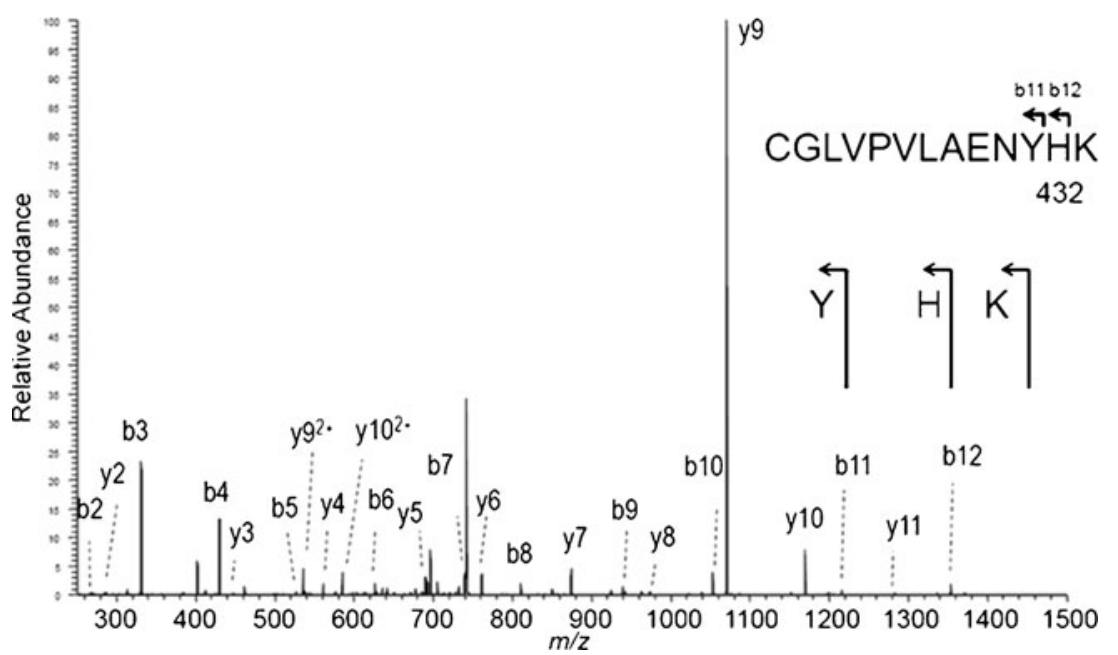


will direct the diagnostics toward CDG-I. These analyses do not require additional serum sampling and provide unambiguous results.

Our findings indicate the potential for mass spectrometric analysis of transferrin (glyco)peptides, not only in CDG-II, but also in the diagnostic track of CDG-I patients. It allowed the detection of two novel mutations in the consensus glycosylation sites, which influence the screening by transferrin IEF.

Acknowledgement This work was supported by the European Commission (LSHM-CT2005-512131, Euroglycanet) and Metakids and a grant from the Ministry of Education, Youth and Sports of the Czech Republic MSM 0021620806.

Open Access This article is distributed under the terms of the Creative Commons Attribution Noncommercial License which permits any noncommercial use, distribution, and reproduction in any medium, provided the original author(s) and source are credited.

\section{References}

Bunkenborg J, Pilch BJ, Podtelejnikov AV, Wisniewski JR (2004) Screening for N-glycosylated proteins by liquid chromatography mass spectrometry. Proteomics 4(2):454-465

Fujita M, Satoh C, Asakawa J, Nagahata Y, Tanaka Y, Hazama R, Krasteff T (1985) Electrophoretic variants of blood proteins in Japanese. VI. Transferrin. Jpn J Hum Genet 30(3):191-200

Guillard M, Morava E, van Delft FL, Hague R, Korner C, Adamowicz M, Wevers RA, Lefeber DJ (2011) Plasma N-Glycan profiling by mass spectrometry for congenital disorders of glycosylation type II. Clin Chem

Hahn SH, Minnich SJ, O'Brien JF (2006) Stabilization of hypoglycosylation in a patient with congenital disorder of glycosylation type Ia. J Inherit Metab Dis 29(1):235-237

Hulsmeier AJ, Paesold-Burda P, Hennet T (2007) N-glycosylation site occupancy in serum glycoproteins using multiple reaction monitoring liquid chromatography-mass spectrometry. Mol Cell Proteomics 6(12):2132-2138

Kamboh MI, Ferrell RE (1987) Human transferrin polymorphism. Hum Hered 37(2):65-81

Lacey JM, Bergen HR, Magera MJ, Naylor S, O'Brien JF (2001) Rapid determination of transferrin isoforms by immunoaffinity liquid chromatography and electrospray mass spectrometry. Clin Chem 47(3):513-518

Marklova E, Albahri Z (2009) Transferrin D protein variants in the diagnosis of congenital disorders of glycosylation (CDG). J Clin Lab Anal 23(2):77-81

Wada Y (2006) Mass spectrometry for congenital disorders of glycosylation, CDG. J Chromatogr B Analyt Technol Biomed Life Sci 838(1):3-8

Wada Y (2007) Mass spectrometry in the detection and diagnosis of congenital disorders of glycosylation. Eur J Mass Spectrom (Chichester, Eng) 13(1):101-103

Wada Y, Nishikawa A, Okamoto N, Inui K, Tsukamoto H, Okada S, Taniguchi N (1992) Structure of serum transferrin in carbohydrate-deficient glycoprotein syndrome. Biochem Biophys Res Commun 189(2):832-836

Wada Y, Gu J, Okamoto N, Inui K (1994) Diagnosis of carbohydratedeficient glycoprotein syndrome by matrix-assisted laser desorption time-of-flight mass spectrometry. Biol Mass Spectrom 23 (2): $108-109$

Yuasa I, Saneshige Y, Suenaga K, Ito K, Gotoh Y (1987) Transferrin variants in Japan and New Zealand. Report of an unusually sialyzed TF variant. Hum Hered 37(1):20-25 